\title{
A GENERALIZED DRAZIN INVERSE
}

\author{
by J. J. KOLIHA
}

(Received 22 May, 1995)

1. Introduction. The main theme of this paper can be described as a study of the Drazin inverse for bounded linear operators in a Banach space $X$ when 0 is an isolated spectral point of the operator. This inverse is useful for instance in the solution of differential equations formulated in a Banach space $X$. Since the elements of $X$ rarely enter into our considerations, the exposition seems to gain in clarity when the operators are regarded as elements of the Banach algebra $L(X)$.

An element $a$ of a complex Banach algebra $A$ is called regular (or relatively regular) if there is $x \in A$ such that axa=a. Relatively regular elements have been extensively studied in the case that $A$ is the Banach algebra $L(X)$ of all bounded linear operators on a complex Banach space $X$; they have been shown to generalize in certain aspects invertible operators.

If $a$ is relatively regular, then it has a generalized inverse, which is an element $b \in A$ satisfying the equations $a b a=a$ and $b a b=b$. (See [18] for a comprehensive account of generalized inverses.) A relation between a relatively regular element and its generalized inverse is reflexive in the sense that if $b$ is a generalized inverse of $a$, then $a$ is a generalized inverse of $b$.

In 1958, Drazin [7] introduced a different kind of a generalized inverse in associative rings and semigroups that does not have the reflexivity property but commutes with the element.

Definition 1.1. Let $a, b$ be elements of a semigroup. An element $b$ is a Drazin inverse of $a$, written $b=a^{d}$, if

$$
a b=b a, \quad b=a b^{2}, \quad a^{k}=a^{k+1} b,
$$

for some nonnegative integer $k$. The least nonnegative integer $k$ for which these equations hold is the Drazin index $i(a)$ of $a$.

The Drazin inverse is an important tool in ring theory (see for instance Hartwig [12]). In spite of not being reflexive, the Drazin inverse is very useful in matrix theory and computations and in various applications of matrices $[4,5,1,21,19]$, primarily because it has a very desirable spectral property. The nonzero eigenvalues of the Drazin inverse are the reciprocals of the nonzero eigenvalues of the given matrix, and the corresponding generalized eigenvectors have the same grade [1, p. 167]. A generalized inverse $a^{\prime}$ of an element $a$ of a complex Banach algebra with the property that the nonzero spectrum of $a^{\prime}$ consists of the reciprocals of the nonzero spectral points of $a$ is often called a spectral inverse [6]. The inverse matrix is a (commuting) spectral inverse.

Caradus [6], King [14] and Lay [16] investigated the Drazin inverse in the setting of bounded linear operators on complex Banach spaces. Caradus [6] proved that a bounded linear operator $T$ on a complex Banach space has a Drazin inverse if and only if 0 is a pole of the resolvent $(\lambda I-T)^{-1}$ of $T$; the order of the pole is equal to the Drazin index of $T$. (See also King [14].) Marek and Zitný [17] discuss the Drazin inverse in detail-for operators as well as for elements of a Banach algebra. Harte [9] associated with each 
quasipolar operator $T$ an operator $T^{\times}$, which is an equivalent of the generalized Drazin inverse studied here. He also investigated quasipolar elements of a normed algebra [10] and extended the concept to this setting. In [11], Harte offered two candidates for quasinilpotent elements in a general associative ring, and introduced the concept of a quasipolar element and its Drazin inverse. Nashed and Zhao [19] investigated the Drazin inverse for closed linear operators and applied it to singular evolution equations and partial differential operators. Drazin [8] investigated extremal definitions of generalized inverses that give a generalization of the original Drazin inverse.

In the theory of the Drazin inverse for matrices $[\mathbf{1}, \mathbf{4}]$ an important role is played by the index of a matrix $A$; this is defined as the least nonnegative integer $k$ for which the nullspaces of $A^{k}$ and $A^{k+1}$ coincide. The index of a matrix coincides with its Drazin index [4].

In operator theory, the concept corresponding to the index of a finite matrix is the ascent (and descent) of a chain-finite bounded linear operator $T[13,22]$. An operator $T$ is chain-finite with the ascent (=descent) $k$ if and only if 0 is a pole of the resolvent $(\lambda I-T)^{-1}$ of order $k$ [13, Proposition 50.2]. If we want to translate results involving the index or the chain-finiteness condition to a Banach algebra $a$ we must interpret the index of $a \in A$ to be 0 if $a$ is invertible, and $k$ if 0 is a pole of $(\lambda e-a)^{-1}$ of order $k$.

2. The Drazin inverse in a ring. In this section $A$ is an associative ring with a unit $e$. First we give an equivalent definition of the Drazin inverse. By $\operatorname{Inv}(A)$ and $N(A)$ we denote the set of all invertible and nilpotent elements in $A$, respectively.

LEMMA 2.1. In a ring $A$ with unit, (1.1) is equivalent to

$$
a b=b a, \quad b=a b^{2}, \quad a-a^{2} b \in N(A) .
$$

The Drazin index $i(a)$ is equal to the nilpotency index of $a-a^{2} b$.

Proof. Suppose that the first two equations in (2.1) hold. Then the element $p=e-a b$ is an idempotent, as $(a b)^{2}=a\left(a b^{2}\right)=a b$. Hence

$$
a^{k}-a^{k+1} b=a^{k} p=(a p)^{k}=(a(e-a b))^{k}=\left(a-a^{2} b\right)^{k},
$$

for any $k \geq 1$, and (1.1) is equivalent to (2.1).

Harte [11] defined quasinilpotent (and quasi-quasinilpotent) elements in an arbitrary associative ring $A$. This concept can be used to define a generalized Drazin inverse in rings.

Definition 2.2. (See [11, Definition 2].) An element $a$ of a ring $A$ is quasinilpotent if, for every $x$ commuting with $a$, we have $e-x a \in \operatorname{Inv}(A)$. The set of all quasinilpotent elements in a ring $A$ will be denoted by $Q N(A)$.

Note that $N(A) \subset Q N(A)$. In a Banach algebra $A$, the preceding definition coincides with the usual definition $\left\|a^{n}\right\|^{1 / n} \rightarrow 0$, which is equivalent to $\lambda e-a \in \operatorname{Inv}(A)$ for all complex $\lambda \neq 0$.

Definition 2.3. Let $a \in A$. An element $b \in A$ is a Drazin inverse $b=a^{D}$ of $a$ if

$$
a b=b a, \quad b=a b^{2}, \quad a-a^{2} b \in Q N(A) .
$$


The Drazin index $i(a)$ of $a$ is the nilpotency index of $a-a^{2} b$ if $a-a^{2} b \in N(A)$, and $i(a)=\infty$ otherwise.

The inverse $a^{d}$ introduced by Definition 1.1 will be called the finite index Drazin inverse.

LEMMA 2.4. In a ring $A$ with unit, an element a has a Drazin inverse $a^{D}$ if and only if there is an idempotent $p$ commuting with a such that

$$
\text { ap } \in Q N(A), \quad a+p \in \operatorname{Inv}(A) .
$$

The Drazin inverse $a^{D}$ is unique and is given by

$$
a^{D}=(a+p)^{-1}(e-p) \text {. }
$$

Proof. Suppose that there is an idempotent $p$ commuting with $a$ and satisfying (2.3). Set $b=(a+p)^{-1}(e-p)$. Then $a b=b a$,

$$
a b=a(a+p)^{-1}(e-p)=(a+p)(a+p)^{-1}(e-p)=e-p
$$

and $a b^{2}=(a b) b=(e-p) b=b(e-p)=b$. Finally,

$$
a-a^{2} b=a(e-a b)=a p \in Q N(A),
$$

so that (2.2) hold.

Conversely, suppose that $b$ satisfies (2.2). Set $p=a-a b$. Since $(a b)^{2}=a\left(a b^{2}\right)=a b, p$ is an idempotent commuting with $a$, and $a p=a-a^{2} b \in Q N(A)$. Further,

$$
(a+p)(b+p)=a b+a p+b p+p=e+a p \in \operatorname{Inv}(A)
$$

as $a p \in Q N(A)$; then also $a+p \in \operatorname{Inv}(A)$. From $(a+p) b=e-p$ it follows that $b=$ $(a+p)^{-1}(e-p)$, which proves the uniqueness of $b$.

We compare our definition of the Drazin inverse with that given by Harte [11] for quasipolar elements of a ring $A$. An element $a$ of a ring $A$ is quasipolar [11, Definition 5] if there exists $b \in A$ such that

$$
b \in \operatorname{comm}^{2}(a), \quad a b=(a b)^{2}, \quad a(e-a b) \in Q N(A) ;
$$

here $\operatorname{comm}^{2}(a)$ denotes the double commutant of $a$. An element $b$ satisfying (2.5) and $b=a b^{2}$ is called a Drazin inverse of $a$ in [11]. Clearly, a Drazin inverse in the sense of Harte is also a Drazin inverse in the sense of Definition 2.3. Conversely however, it cannot be guaranteed that the Drazin inverse $b=a^{D}$ defined according to Definition 2.3 is in the double commutant of $a$. If $A$ is not only a ring, but a Banach algebra, this fact follows from topological and spectral considerations discussed in the next section; (see also [11, Theorem 7.5.3]).

3. Isolated spectral points. From this point on, $A$ denotes a complex unital Banach algebra with unit $e$; $\operatorname{Inv}(A), Q N(A)$ and $N(A)$ denote the set of all invertible elements of $A$, the set of all quasinilpotent elements of $A$ and the set of all nilpotent elements of $A$, respectively. For each $a \in A, \sigma(a)$ denotes the spectrum of $a, \rho(a)$ the resolvent set of $a$ and $r(a)$ the spectral radius of $a$. We write iso $\sigma(a)$ for the set of all isolated spectral points of $a$ and acc $\sigma(a)$ for the set of all accumulation points of $\sigma(a)$. By $H(a)$ we denote the set of all complex valued functions $f$, each defined and holomorphic in an open 
neighbourhood $\Delta(f)$ of $\sigma(a)$. If $\Delta$ is an open subset of the complex plane and $K$ a compact subset of $\Delta$, we define a cycle $\gamma$ to be a $(\Delta, K)$-cycle if $\gamma$ lies in $\Delta \backslash K$, ind $(\gamma ; \lambda)=0$ for every $\lambda \notin \Delta$, and ind $(\gamma ; \lambda)=1$ for every $\lambda \in K$. The functional calculus for $a \in A$ is defined for any function $f \in H(a)$ by

$$
f(a)=\frac{1}{2 \pi i} \int_{\gamma} f(\lambda)(\lambda e-a)^{-1} d \lambda,
$$

where $\gamma$ is a $(\Delta(f), \sigma(a))$-cycle. We recall that, in commutative Banach algebras, the spectral radius is submultiplicative, subadditive and continuous. This fact will be used throughout the paper usually without a further comment. For an element $a \in A$ we define the spectral index $s$-ind( $a$ ) to be 0 if $a$ is invertible and $k$ if 0 is a pole of order $k$ of the resolvent $(\lambda e-a)^{-1}$. (We do not use the word "index" because in operator theory it is reserved for the difference $\operatorname{dim} T^{-1}(0)-\operatorname{codim} T(X)$, where $T$ is a bounded linear operator on a Banach space $X[13]$.) For spectral theory and functional calculus in Banach algebras see Heuser [13, Chapter VII].

We start with a characterization of isolated spectral points of an element of $A$ that will provide the main tool for the development of the generalized Drazin inverse. The result is a special case of a theorem obtained by the author [15].

TheOREM 3.1. Let $a \in A$. Then $0 \notin \operatorname{acc} \sigma(a)$ if and only if there is an idempotent $p \in A$ commuting with $a$ and such that

$$
a p \in Q N(A), \quad p+a \in \operatorname{Inv}(A) .
$$

Moreover, $0 \in$ iso $\sigma(a)$ if and only if $p \neq 0$, in which case $p$ is the spectral idempotent of a corresponding to $\lambda=0$.

Proof. Clearly, $a \in A$ is invertible if and only if (3.1) holds with $p=0$.

Let $0 \in$ iso $\sigma(a)$. The spectral idempotent $p$ of $a$ is defined by $p=f(a)$, where $f \in H(a)$ is such that $f=1$ in a neighbourhood of 0 and $f=0$ in a neighbourhood of $\sigma(a) \backslash\{0\}$. Then $p^{2}=p \neq 0, p$ commutes with $a$, and $a p=h(a)$, where $h(\lambda)=\lambda f(\lambda)$; as $\sigma(a p)=\sigma(h(a))=h(\sigma(a))=\{0\}$ by the spectral mapping theorem, ap $\in Q N(A)$. The function $g$ defined by $g(\lambda)=f(\lambda)+\lambda$ is in $H(a)$ and is nonzero on the spectrum of $a$; so $g(a)=p+a \in \operatorname{Inv}(A)$.

Conversely, assume that there is a nonzero idempotent $p$ commuting with $a$ such that (3.1) hold. For any $\lambda$, we have

$$
\lambda e-a=(\lambda e-a p) p+(\lambda e-(p+a))(e-p) .
$$

There is $r>0$ (e.g. $\left.r=\left\|(p+a)^{-1}\right\|^{-1}\right)$ such that $\lambda e-(p+a) \in \operatorname{Inv}(A)$ if $|\lambda|<r$. Since $a p \in Q N(A), \lambda e-a p \in \operatorname{Inv}(A)$, for all $\lambda \neq 0$. Hence

$$
(\lambda e-a)^{-1}=(\lambda e-a p)^{-1} p+(\lambda e-(p+a))^{-1}(e-p),
$$

whenever $0<|\lambda|<r$. Since $p \neq 0,0 \in$ iso $\sigma(a)$. To show that $p$ is the spectral idempotent 
of $a$ corresponding to $a$, we pick a function $f \in H(a)$ with $f=1$ in a neighbourhood of 0 and $f=0$ in a neighbourhood of $\sigma(a) \backslash\{0\}$. By (3.2),

$$
\begin{aligned}
f(a) & =\frac{1}{2 \pi i} \int_{\gamma}(\lambda e-a)^{-1} d \lambda \\
& =\frac{1}{2 \pi i} \int_{\gamma}(\lambda e-a p)^{-1} p d \lambda+\frac{1}{2 \pi i} \int_{\gamma}(\lambda e-(p+a))^{-1}(e-p) d \lambda \\
& =\frac{1}{2 \pi i} \int_{\gamma} \sum_{n=0}^{\infty} \lambda^{-n-1} a^{n} p+0=p,
\end{aligned}
$$

where $\gamma$ is a $(\{\lambda:|\lambda|<r\},\{0\})$-cycle.

Harte [10] obtained a similar result using the concept of a quasipolar element in a Banach algebra. An element $a$ of a Banach algebra $A$ is quasipolar [10, p. 257] if there is an idempotent $q \in A$ commuting with $a$ such that

$$
a(e-q) \in Q N(A), \quad q \in(A a) \cap(a A) .
$$

We give a proof using the preceding characterization of isolated spectral points.

Theorem 3.2. (Harte [10, Theorem 9.7.6].) An element $a \in A$ is quasipolar if and only if $0 \notin \operatorname{acc} \sigma(a)$.

Proof. Suppose that $a$ is quasipolar, satisfying (3.3). Write $p=e-q$. Then there are $u, v \in A$ such that $e-p=u a=a v$. A simple calculation shows that

$$
(a+p)(u a v+p)=e+a p=(u a v+p)(a+p) .
$$

Since $e+a p \in \operatorname{Inv}(A)$, we have $a+p \in \operatorname{Inv}(A)$. Then $0 \notin \operatorname{acc} \sigma(a)$ by the preceding theorem.

Conversely, if $0 \notin \operatorname{acc} \sigma(a)$, the conclusions of the preceding theorem hold. Since $(a+p)(e-p)=a(e-p)$, we have

$$
a(a+p)^{-1}(e-p)=e-p=(a+p)^{-1}(e-p) a .
$$

Then (3.3) holds for $q=e-p$.

NoTE 3.3. It is often useful to know that (3.1) can be replaced by

$$
a p \in Q N(A), \quad a+\alpha p \in \operatorname{Inv}(A), \text { for some } \alpha \neq 0 .
$$

Changes to the preceding proof are obvious.

NoTE 3.4. In view of the Laurent expansion for the resolvent $(\lambda e-a)^{-1}$, Theorem 3.1 gives the following criterion: 0 is a pole of $(\lambda e-a)^{-1}$ if and only if there is a nonzero idempotent $p$ commuting with $a$ such that

$$
a p \in N(A), \quad a+p \in \operatorname{Inv}(A) .
$$

4. The Drazin inverse in a Banach algebra. In this section $A$ is a complex unital Banach algebra. By $\operatorname{Inv}(A)$ and $Q N(A)$ we denote the sets of all invertible and quasinilpotent elements of $A$, respectively. By definition, an element $x$ of a Banach 
algebra $A$ is quasinilpotent if $\left\|x^{n}\right\|^{1 / n} \rightarrow 0$. As mentioned earlier, this is equivalent to the Harte definition of a quasinilpotent (Definition 2.2).

Definition 4.1. Let $a \in A$. An element $b \in A$ is called a Drazin inverse of $a$, and is denoted by $a^{D}$, if it satisfies the conditions

$$
a b=b a, \quad b=a b^{2}, \quad a-a^{2} b \in Q N(A) .
$$

If $0 \in \rho(a)$, that is, if $a$ is invertible, then $a^{D}=a^{-1}$. Even though the defining relations (4.1) in a Banach algebra are the same as the defining relations (2.1) in a ring, topology enters here via the definition of quasinilpotents which is dependent on the norm of $A$. In particular, we shall see that in a Banach algebra the Drazin inverse of $a$ is in the double commutant of $a$; this is not always the case in a ring.

THEOREM 4.2. The following conditions on an element $a \in A$ are equivalent:

(i) $0 \notin$ acc $\sigma(a)$,

(ii) there is an idempotent $p \in A$ commuting with a satisfying (3.1),

(iii) a has a Drazin inverse.

In this case the Drazin inverse is unique, and is given by

$$
a^{D}=(a+p)^{-1}(e-p)
$$

where $p$ is the spectral idempotent of a corresponding to 0 .

Proof. Conditions (i) and (ii) are equivalent by Theorem 3.1, and (ii) and (iii) are equivalent by Lemma 2.4. The explicit formula (4.2) was also obtained in Lemma 2.4.

Harte obtained the following result, defining in effect a Drazin inverse $a^{\times}$for a quasipolar element $a \in A$. (See the definition following Theorem 3.1.) We give an alternative proof based on our definition of the Drazin inverse.

THEOREM 4.3. Harte [10, Theorem 7.5.3]. If $a \in A$ is quasipolar, then there are unique elements $\dot{a}, a^{\times} \in A$ such that

$$
(e-\dot{a}) a=a(e-\dot{a}) \in Q N(A), \quad a^{\times} a=a a^{\times}=\dot{a}=\dot{a}^{2}, \quad a^{\times} \dot{a}=\dot{a} a^{\times}=a^{\times} .
$$

Proof. A quasipolar element $a$ satisfies $0 \notin \operatorname{acc} \sigma(a)$ (Theorem 3.2). Let $p$ be the spectral idempotent of $a$ at $\lambda=0$, and define $a=e-p, a^{\times}=a^{D}$ which exists by the preceding theorem. (4.3) is then verified using Theorems 3.1 and 4.2, which proves the existence. Further, if $\dot{a}$ and $a^{\times}$satisfy (4.3), then

$$
a^{\times}-a\left(a^{\times}\right)^{2}=a^{\times}\left(e-a a^{\times}\right)=a^{\times}(e-\dot{a})=0,
$$

and

$$
a-a^{2} a^{\times}=a\left(e-a a^{\times}\right)=a(e-\dot{a}) \in Q N(A) .
$$

Then $a^{\times}=a^{D}$ and $\dot{a}=a a^{D}=e-p$, which proves the uniqueness.

The explicit formula (4.2) was given for matrices by Rothblum [20] in the form $a^{d}=(a-p)^{-1}(e-p)$. It can be deduced from the next theorem that $a^{D}$ satisfies a more general equation

$$
a^{D}=(a+\alpha p)^{-1}(e-p), \quad \alpha \neq 0 .
$$

Theorem 4.2 shows that, in the case that 0 is a pole rather than just an isolated spectral 
point of $(\lambda e-a)^{-1}$, the Drazin inverse $a^{D}$ reduces to the finite index Drazin inverse $a^{d}$. In this case, the Drazin index of $a$ is given by

$$
i(a)=s-\operatorname{ind}(a)
$$

The next result generalizes [17, Theorem 13.4]. It shows that $a^{D}$ is a spectral inverse of $a$ that lies in the double commutant of $a$.

TheOREM 4.4. If $0 \in$ iso $\sigma(a)$, then

$$
a^{D}=f(a)
$$

where $f \in H(a)$ is such that $f$ is 0 in a neighbourhood of 0 and $f(\lambda)=\lambda^{-1}$ in $a$ neighbourhood of $\sigma(a) \backslash\{0\}$. Hence $a^{D}$ commutes with every element of $A$ that commutes with $a$, and

$$
\sigma\left(a^{D}\right) \backslash\{0\}=\left\{\lambda^{-1}: \lambda \in \sigma(a) \backslash\{0\}\right\} .
$$

Proof. Write the spectral idempotent $p$ of $a$ at 0 as $p=g(a)$, where $g \in H(a), g$ is 1 in a neighbourhood of $0, g$ is 0 in a neighbourhood of $\sigma(a) \backslash\{0\}$, and set

$$
f(\lambda)=(\lambda+g(\lambda))^{-1}(1-g(\lambda)) \text {. }
$$

By (4.2), $a^{D}=(a+p)^{-1}(e-p)=f(a)$; it is easy to check that $f$ has the required property. Equality (4.5) follows from the spectral mapping theorem.

5. Properties of the Drazin inverse. We start with a version of the Laurent series for the resolvent of an element $a$ of a Banach algebra $A$ in a neighbourhood of an isolated spectral point 0 . The theorem generalizes a result of Caradus [6]. This was obtained for operators under the additional assumption that 0 is a pole of the operator resolvent.

THEOREM 5.1. Let $0 \in$ iso $\sigma(a)$, and let $b$ be the Drazin inverse of $a$. Then, on some punctured disc $\{\lambda: 0<|\lambda|<r\}$, we have

$$
(\lambda e-a)^{-1}=\sum_{n=1}^{\infty} \lambda^{-n} a^{n-1}(e-a b)-\sum_{n=0}^{\infty} \lambda^{n} b^{n+1} .
$$

Proof. Let $p$ be the spectral idempotent of $a$ corresponding to $\lambda=0$; then $b=(a+p)^{-1}(e-p)$, by (4.2). In some disc $\{\lambda:|\lambda|<r\}, \lambda e-(a+p)$ is invertible; also ap is quasinilpotent. According to (3.2), if $0<|\lambda|<r$, then

$$
\begin{aligned}
(\lambda e-a)^{-1} & =(\lambda e-a p)^{-1} p+(\lambda e-(a+p))^{-1}(e-p) \\
& =\sum_{n=1}^{\infty} \lambda^{-n} a^{n-1} p-\sum_{n=0}^{\infty} \lambda^{n}(a+p)^{-n-1}(e-p) \\
& =\sum_{n=1}^{\infty} \lambda^{-n} a^{n-1}(e-a b)-\sum_{n=0}^{\infty} \lambda^{n} b^{n+1} .
\end{aligned}
$$

THEOREM 5.2. Let $b=a^{D}$ be the Drazin inverse of an element $a \notin \operatorname{Inv}(A)$. Then $s$-ind $(b)=1$. 
Proof. The element $p=e-a b$ is the (nonzero) spectral idempotent of $a$ corresponding to $\lambda=0$ with $a p \in Q N(A)$ and $a+p \in \operatorname{Inv}(A)$. Then

$$
b p=b(e-a b)=b-a b^{2}=0
$$

and

$$
(a+p)(b+p)=a b+a p+p=e+a p \in \operatorname{Inv}(A),
$$

so that $b+p \in \operatorname{Inv}(A)$. By Theorem $3.1,0 \in$ iso $\sigma(b)$ with the spectral idempotent $p$; since $b p=0, \lambda=0$ is a simple pole of $(\lambda e-b)^{-1}$.

TheOREM 5.3. Let $a \notin \operatorname{Inv}(A)$. Then $\left(a^{D}\right)^{D}=A$ if and only if $s$-ind $(a)=1$.

Proof. Suppose that $b=a^{D}$ and that 0 is a simple pole of $(\lambda e-a)^{-1}$ with the spectral idempotent $p$. Then $a-a^{2} b=a(e-a b)=a p=0$, and

$$
a b=b a, \quad b-a b^{2}=0, \quad a-a^{2} b=0 .
$$

This shows that $b^{D}=a$. Conversely, if $a^{D}=b$ and $b^{D}=a$, then the preceding equations hold and signify that 0 is a simple pole of $(\lambda e-a)^{-1}$.

In the case that 0 is a pole of $(\lambda e-a)^{-1}$, the Drazin inverse $a^{D}$ and the finite index Drazin inverse $a^{d}$ of $a$ coincide; if 0 is a simple pole, $a^{d}$ is then called the group inverse of $a$, and in matrix theory it is usually denoted by $a^{*}$. The name was chosen because the elements $a$ and $a^{*}$ generate a multiplicative Abelian group in $A$; the idempotent $a a^{\#}$ plays the role of the group identity.

The properties of the Drazin inverse stated in the following theorem are easily verified by using Theorems 3.1, 4.2 and 4.4 .

THEOREM 5.4. Suppose that $a \in A$ has the Drazin inverse $a^{D}$ and that $p$ is the spectral idempotent of a corresponding to 0 . Then

(i) $\left(a^{n}\right)^{D}=\left(a^{D}\right)^{n}$ for all $n=1,2, \ldots$,

(ii) $\left(a^{D}\right)^{D}=a^{2} a^{D}=a(e-p)$,

(iii) $\left(\left(a^{D}\right)^{D}\right)^{D}=a^{D}$,

(iv) $a^{D}\left(a^{D}\right)^{D}=a a^{D}=e-p$.

The next result deals with the inverse of a product, and gives an indication of the usefulness of the Drazin inverse as it provides a short proof of the fact that 0 is an isolated spectral point of $a b$ if $a, b$ commute and 0 is an isolated spectral point for both $a$ and $b$. The result is well known for matrices [4, Theorem 7.8.4].

THEOREM 5.5. Let $a, b$ be commuting elements of $A$ such that $a^{D}$ and $b^{D}$ exist. Then $(a b)^{D}$ exists and

$$
(a b)^{D}=a^{D} b^{D}
$$

Proof. The elements $a, b, a^{D}, b^{D}$ all commute. Then

$$
a b\left(a^{D} b^{D}\right)^{2}=a\left(a^{D}\right)^{2} b\left(b^{D}\right)^{2}=a^{D} b^{D} .
$$

Further,

$$
a b-(a b)^{2} a^{D} b^{D}=\left(a-a^{2} a^{D}\right)\left(b-b^{2} b^{D}\right)+a^{2} a^{D}\left(b-b^{2} b^{D}\right)+b^{2} b^{D}\left(a-a^{2} a^{D}\right),
$$


and $r\left(a b-(a b)^{2} a^{D} b^{D}\right)=0$, as the spectral radius is subadditive and submultiplicative in commutative subalgebras of $A$. Hence $a b-(a b)^{2} a^{D} b^{D} \in Q N(A)$.

This result is implicit in Harte [10, Theorem 7.5.4], where it is proved that the product of commuting quasipolar elements is quasipolar.

The proof of the preceding theorem can be adapted to show that the product of two commuting elements of a Banach algebra with a finite Drazin index has a finite index Drazin inverse. Indeed, it is enough to observe that the product of commuting elements, one of which is nilpotent, is also nilpotent, and the sum of commuting nilpotent elements is nilpotent.

Bouldin [2] gives an example of two noncommuting bounded linear operators $A, B$ on a complex Banach space that have the finite index Drazin inverses $A^{d}, B^{d}$, but whose product $A B$ is not Drazin invertible [2, Example 4.1]. Bouldin also gives some conditions under which the equation $(A B)^{d}=B^{d} A^{d}$ holds in the noncommutative case.

THEOREM 5.6. If $a^{D}$ exists, $b \in Q N(A)$ and $a b=b a$, then also $(a+b)^{D}$ exists and

$$
(a+b)^{D}=(a+b+p)^{-1}(e-p)
$$

where $p$ is the spectral idempotent of $a$ at $\lambda=0$.

Proof. Let $p$ be the spectral idempotent of $a$ at $\lambda=0$; the set $\{a, b, p\}$ is commutative, $a+p \in \operatorname{Inv}(A)$ and $a p \in Q N(A)$. Then

$$
a+b+p=(a+p)+b \in \operatorname{Inv}(A), \quad(a+b) p=a p+b p \in Q N(A) .
$$

Hence $0 \notin$ acc $\sigma(a+b)$, by Theorem 3.1 and the explicit formula for the Drazin inverse of $a+b$ follows from (4.2).

The preceding result shows that if $0 \in$ iso $\sigma(a), b \in Q N(A)$ and $a b=b a$, then $0 \in$ iso $\sigma(a+b)$. This is implicitly contained in Harte [10, Theorem 7.5.4] formulated in terms of quasipolarity.

TheOREM 5.7. If $a^{D}, b^{D}$ exist and $a b=b a=0$, then also $(a+b)^{D}$ exists and

$$
(a+b)^{D}=a^{D}+b^{D} \text {. }
$$

Proof. As in [7] we observe that $a, b, a^{D}, b^{D}$ all commute, that $a b^{D}=a b\left(b^{D}\right)^{2}=0$ and $a^{D} b=a b\left(a^{D}\right)^{2}=0$. Hence

$$
(a+b)\left(a^{D}+b^{D}\right)^{2}=a\left(a^{D}\right)^{2}+b\left(b^{D}\right)^{2}=a^{D}+b^{D},
$$

and

$$
(a+b)-(a+b)^{2}\left(a^{D}+b^{D}\right)=\left(a-a^{2} a^{D}\right)+\left(b-b^{2} b^{D}\right) \in Q N(A)
$$

which shows that $(a+b)^{D}=a^{D}+b^{D}$.

6. Representations of the Drazin inverse. From the Laurent expansion we get a limit representation of the Drazin inverse in a Banach algebra $A$.

THEOREM 6.1. Let $0 \in$ iso $\sigma(a)$ with the corresponding spectral idempotent $p$. Then

$$
a^{D}=\lim _{\lambda \rightarrow 0}(a-\lambda e)^{-1}(e-p)
$$


Proof. If we multiply both sides of (5.1) by $e-p$, we get

$$
(\lambda e-a)^{-1}(e-p)=-\sum_{n=0}^{\infty} \lambda^{n} b^{n+1}
$$

where $b=a^{D}$. The result then follows.

We give a representation of the Drazin inverse in two special cases. For the first case we recall that much of the elementary spectral theory in a Banach algebra $A$ depends on the expansion

$$
(e-a)^{-1}=\sum_{n=0}^{\infty} a^{n},
$$

valid in the case that $a^{n} \rightarrow 0$. There is a generalization of this expansion in the case that $a^{n} \rightarrow p$, where $p$ need not be 0 .

THEOREM 6.2. Let $a^{n} \rightarrow p$ in $A$. Then $s$-ind $(e-a) \leq 1$ and

$$
(e-a)^{D}=\sum_{n=0}^{\infty} a^{n}(e-p) .
$$

Proof. We observe that $p^{2}=p$; if $c$ is defined by $c=a-p$, then $c^{n}=(a-p)^{n}=$ $a^{n}-p \rightarrow 0$, and $c p=p c=0$. Further, $p$ is an idempotent commuting with $e-a$, such that $(e-a) p=0$ and $e-a+p=e-c \in \operatorname{Inv}(A)$; by Theorem 3.1, $\lambda=0$ is a resolvent point of $e-a$ or a simple pole of its resolvent. As $a^{n}(e-p)=\left(c^{n}-p\right)(e-p)=c^{n}(e-p)$,

$$
\begin{aligned}
\sum_{n=0}^{\infty} a^{n}(e-p) & =\sum_{n=0}^{\infty} c^{n}(e-p)=(e-c)^{-1}(e-p) \\
& =(e-a+p)^{-1}(e-p)=(e-a)^{D} .
\end{aligned}
$$

For the second case note that $\exp (t a) \rightarrow 0($ as $t \rightarrow \infty)$ implies

$$
a^{-1}=-\int_{0}^{\infty} \exp (t a) d t
$$

This generalizes to elements $a$ for which $\exp (t a)$ converges, but not necessarily to 0 .

THEOREM 6.3. Let $\exp (t a) \rightarrow p$ as $t \rightarrow \infty$. Then $s-\operatorname{ind}(a) \leq 1$, and

$$
a^{D}=-\int_{0}^{\infty} \exp (t a)(e-p) d t
$$

Proof. We have $p^{2}=p$. If $c=a-p$, then

$$
\exp (t c)=\exp (t a) \exp (-t p)=\exp (t a)\left(e-p+e^{-t} p\right) \rightarrow p(e-p)=0
$$

hence $\sigma(c)$ lies in the left half-plane, and $c$ is invertible. Further,

$$
a p=a \lim _{s \rightarrow \infty} \frac{1}{S} \int_{0}^{s} \exp (t a) d t=\lim _{s \rightarrow \infty}(\exp (s a)-e)=0,
$$

which implies that $p c=c p=-p$. To conclude that $0 \notin \operatorname{acc} \sigma(a)$, it is enough to observe that $a-p=c \in \operatorname{Inv}(A)$ (Note 3.3). 
Finally, since $\exp (t a)(e-p)=\exp (t c)(e-p)$ and since $\exp (t c) \rightarrow 0$ as $t \rightarrow \infty$, we have

$$
\begin{aligned}
\int_{0}^{\infty} \exp (t a)(e-p) d t & =\int_{0}^{\infty} \exp (t c)(e-p) d t=(e-p) \int_{0}^{\infty} \exp (t c) d t \\
& =-c^{-1}(e-p)=-(a-p)^{-1}(e-p) \\
& =-a^{D}
\end{aligned}
$$

by (4.4).

The following theorem generalizes the "core-nilpotent" decomposition $A=A(I-$ $P)+A P$ of a square matrix $[1$, p. 175] to elements of a Banach algebra. An operator version was given by King [14] in the special case that 0 is a pole of the operator resolvent.

THEOREM 6.4. Let $a \in A$. Then $0 \in$ iso $\sigma(a)$ if and only if there are $x, y \in A$ such that

$$
a=x+y, \quad x y=y x=0, \quad s \text {-ind }(x)=1, \quad y \in Q N(A) .
$$

Such a decomposition is unique.

Proof. If (6.4) hold, then $a^{D}=(x+y)^{D}=x^{D}+y^{D}=x^{D}$, by Theorem 5.7, and so $0 \in$ iso $\sigma(a)$, by Theorem 4.2. The decomposition is unique as $x=\left(x^{D}\right)^{D}=\left(a^{D}\right)^{D}$, by Theorem 5.3.

Conversely, let 0 be an isolated spectral point of $a$ with the corresponding spectral idempotent $p$, and let

$$
x=a(e-p), \quad y=a p .
$$

Then $y=a p \in Q N(A), x p=0$ and $x+p=(a+p)-a p \in \operatorname{Inv}(A)$ (invertible minus quasinilpotent). The last two conditions imply that $s$-ind $(x)=1$.

The preceding result shows that the calculation of the Drazin inverse can be reduced to the calculation of the finite index Drazin inverse, in fact of the group inverse, as

$$
a^{D}=(a(e-p))^{\sharp} \text {. }
$$

Thus we have a complete description of elements which possess a Drazin inverse.

COROllary 6.5. An element a of a Banach algebra $A$ has the Drazin inverse $a^{D}$ if and only if it is the sum of an element $x$ with a finite Drazin index and a quasinilpotent element $y$ such that $x y=y x=0$. Then

$$
a^{D}=x^{d}
$$

In addition, $a^{D}=a^{d}$ if and only if $y$ is nilpotent.

7. The Drazin inverse for bounded linear operators. Let $X$ be a complex Banach space and $L(X)$ the Banach algebra of all bounded linear operators on $X$ with the operator norm

$$
\|A\|=\sup _{\|x\|=1}\|A x\|
$$


We write $N(A)$ for the null space of $A \in L(X)$, and $R(A)$ for its range. The following result was obtained by Lay [16] for the finite index Drazin inverse.

THEOREM 7.1. Let $A \in L(X)$ be such that $0 \notin$ acc $\sigma(A)$. If $A^{D}$ is the Drazin inverse of $A$ as an element of the algebra $L(X)$, then $X=R\left(A^{D} A\right) \oplus N\left(A^{D} A\right), A=A_{1} \oplus A_{2}$ with respect to this direct sum and

$$
A^{D}=A_{1}^{-1} \oplus 0 .
$$

Proof. Let $P$ be the corresponding spectral idempotent, so that $P=I-A^{D} A$, and $X=N(P) \oplus R(P)=R\left(A^{D} A\right) \oplus N\left(A^{D} A\right)$. From Theorem 3.1 we can deduce that $A=$ $A_{1} \oplus A_{2}$ with $A_{1}$ invertible in $L\left(R\left(A^{D} A\right)\right)$ and $A_{2}$ quasinilpotent in $L\left(N\left(A^{D} A\right)\right)$. Observe that $P=0 \oplus I$. Then by (4.2)

$$
A^{D}=(A+P)^{-1}(I-P)=\left(A_{1} \oplus\left(A_{2}+I\right)\right)^{-1}(I \oplus 0)=A_{1}^{-1} \oplus 0 .
$$

Campbell [3] gives an alternative generalization of the Drazin inverse of bounded linear operators. Suppose that $A \in L(X)$ is such that the hyper-range

$$
R\left(A^{\infty}\right)=\bigcap_{n=0}^{\infty} R\left(A^{n}\right)
$$

is closed and complemented by a closed $A$-invariant subspace $M$. Then

$$
A=U \oplus V
$$

with respect to the decomposition $X=R\left(A^{\infty}\right) \oplus M$. If $U$ is invertible, Campbell defines

$$
A^{\delta}=U^{-1} \oplus 0 \text {. }
$$

Then

$$
A A^{\delta}=A^{\delta} A, \quad A^{\delta}=A\left(A^{\delta}\right)^{2}, \quad A-A^{2} A^{\delta}=0 \oplus V .
$$

$A^{\delta}$ may exist when $0 \notin$ iso $\sigma(A)$; in this case $A^{\delta}$ need not be unique [3, Example 3].

If 0 is a pole of $(\lambda I-A)^{-1}$, then $R\left(A^{D} A\right)=R\left(A^{k}\right)=R\left(A^{\infty}\right)$ for $k=s$-ind $(A)$. In this case the inverses $A^{\delta}, A^{D}$ and $A^{d}$ all exist and are equal.

If 0 is an essential singularity of $(\lambda I-A)^{-1}$, then $A^{D}$ exists, but $A^{\delta}$ need not. If, in addition, $R\left(A^{\infty}\right)=R\left(A^{D} A\right)$, then $A^{\delta}$ exists and $A^{\delta}=\left(A_{1} \oplus A_{2}\right)^{\delta}=A_{1}^{-1} \oplus 0=A^{D}$.

From (7.1) we see that if $A^{\delta}$ exists and $V$ is quasinilpotent, then $0 \in$ iso $\sigma(A)$ and $A^{D}=A^{\delta}$. If $A^{D}$ and $A^{\delta}$ both exist and are equal, then $V$ in (7.1) is quasinilpotent. Note that $R\left(A^{\infty}\right)=R\left(A^{D} A\right)$ whenever $A^{D}=A^{\delta}$.

We close the section with an application to stability of differential equations in Banach spaces that generalizes a result stated in [17, p. 111].

THEOREM 7.2. Suppose $A \in L(X)$ is such that $\left(A^{n}\right)$ converges, and let

Then the differential equation

$$
B=(I+A)(A-I)^{D} \text {. }
$$

$$
\dot{x}(t)=B x(t), \quad x(0)=u \quad(t \geq 0, u \in X)
$$

has a steady state solution as $t \rightarrow \infty$; that is, $\lim _{t \rightarrow \infty} x(t)$ exists.

Proof. In the proof of Theorem 6.2 we established that the limit $P$ of $\left(A^{n}\right)$ is the 
spectral idempotent of $A$ corresponding to $\lambda=1$. Write $P=g(A)$, where $g \in H(A)$ is such that $g=1$ in a neighbourhood of 1 and $g=0$ in a neighbourhood of $\sigma(A) \backslash\{1\}$. Define also $f \in H(A)$ such that $f$ is equal to 0 in a neighbourhood of 1 and to $(1+\lambda) /(\lambda-1)$ in a neighbourhood of $\sigma(A) \backslash\{1\}$. Then $B=f(A)$. We show that

$$
B P=0, \quad B+P \in \operatorname{Inv}(L(X)) \text {. }
$$

Indeed, $B P=f(A) g(A)=(f g)(A)=0$, as $f g=0$. Also, $f+g \neq 0$ on $\sigma(A)$, so that $B+P=f(A)+g(A)$ is invertible. This ensures that 0 is a simple pole of the resolvent of $B$. The function $f$ maps the open unit disc $D$ onto the open left half plane $H$ of $\mathbf{C}$. Since $\sigma(A) \backslash\{1\} \subset D$, the spectral mapping theorem implies that

$$
\sigma(B) \backslash\{0\}=f(\sigma(A) \backslash\{1\}) \subset f(D) \subset H .
$$

The condition $\sigma(B) \backslash\{0\} \subset H$ together with $\sigma$-ind $(B) \leqslant 1$ guarantees that $\exp (s B)$ converges as $s \rightarrow \infty$. This proves the result, as the solution to the differential equation above is given by $x(t)=\exp (t B) u$.

\section{Examples.}

EXAmple 8.1. Define an operator $B$ on the Banach space $l^{1}$ by the infinite matrix

$$
\left[\begin{array}{ccccc}
0 & 0 & 0 & 0 & \ldots \\
1 & 0 & 0 & 0 & \ldots \\
0 & \frac{1}{2} & 0 & 0 & \ldots \\
0 & 0 & \frac{1}{3} & 0 & \ldots \\
0 & 0 & 0 & \frac{1}{4} & \ldots \\
\ldots & \ldots & \ldots & & \ldots
\end{array}\right]
$$

Then $B$ is a quasinilpotent (but not nilpotent) element of the Banach algebra $L\left(l^{1}\right)$ of all bounded linear operators on $l^{1}\left[22\right.$, p. 280]. The finite index Drazin inverse $B^{d}$ does not exist; the Drazin inverse $B^{D}$ is the zero operator. matrix

Example 8.2. Define a diagonal operator $A$ on the Banach space $l^{1}$ by the infinite

$$
\left[\begin{array}{ccccc}
0 & 0 & 0 & 0 & \ldots \\
0 & \alpha_{2} & 0 & 0 & \ldots \\
0 & 0 & \alpha_{3} & 0 & \ldots \\
0 & 0 & 0 & \alpha_{4} & \ldots \\
\ldots & & \ldots & & \ldots
\end{array}\right]
$$

where $0<\varepsilon \leq\left|\alpha_{n}\right| \leq M$, for all $n=2,3, \ldots$ Then $A$ is an element of the Banach algebra $L\left(l^{1}\right)$ with the spectrum $\sigma(A)=\{0\} \cup \operatorname{cl}\left\{\alpha_{n}: n=2,3, \ldots\right\}$, and, for any $f \in H(A)$,

$$
f(A)\left(\xi_{n}\right)=\sum_{n=2}^{\infty} \alpha_{n} f\left(\xi_{n}\right) e_{n}
$$

where $e_{1}, e_{2}, \ldots$ is the standard Schauder basis in $l^{1}$ [22]. It follows that 0 is a simple pole of $A$ with the spectral idempotent $P\left(\xi_{n}\right)=\xi_{1} e_{1}$. By Theorem 4.4, the inverse $A^{D}=A^{*}$ is 
given by $A^{D}=f(A)$, where $f(\lambda)$ is 0 in a neighbourhood of 0 and $\lambda^{-1}$ in a neighbourhood of $\sigma(A) \backslash\{0\}$; hence $A^{D}$ is defined by the matrix

$$
\left[\begin{array}{ccccc}
0 & 0 & 0 & 0 & \ldots \\
0 & \alpha_{2}^{-1} & 0 & 0 & \ldots \\
0 & 0 & \alpha_{3}^{-1} & 0 & \ldots \\
0 & 0 & 0 & \alpha_{4}^{-1} & \ldots \\
\ldots & & \ldots & & \ldots
\end{array}\right]
$$

Example 8.3. Let $X=l^{1} \oplus l^{1}$ and let $T=A \oplus B$, where $A$ and $B$ are defined in the preceding two examples. Set $U=A \oplus 0$ and $V=0 \oplus B$; then $T, U, V \in L(X)$ and

$$
T=U+V, \quad U V=V U=0 .
$$

$U$ has a simple pole at 0 and $V$ is quasinilpotent in $L(X)$.

The spectrum of $T$ is $\sigma(T)=\sigma(A) \cup \sigma(B)=\sigma(A)$. See [22, p. 289]. By Theorem 6.4, $T$ has an isolated spectral point at 0 , which is an essential singularity. Also $T$ has the Drazin inverse $T^{D}$ but not the finite index Drazin inverse $T^{d}$. We have

$$
T^{D}=U^{d}=A^{\#} \oplus 0
$$

EXAMPLE 8.4. Let $A$ be the Banach algebra $C[0,1]$ of all continuous complex valued functions on $[0,1]$ equipped with the supremum norm. The zero element 0 has the Drazin inverse $0^{D}=0$. Every ball centred at 0 contains an element $x(t)=\varepsilon t$ for a sufficiently small $\varepsilon>0$. Since $\sigma(x)=x([0,1])=[0, \varepsilon], 0 \notin$ iso $\sigma(a)$ and $x^{D}$ does not exist. This shows that the set of all elements of a Banach algebra which possess a Drazin inverse is not necessarily open in the algebra.

\section{REFERENCES}

1. A. Ben-Israel and T. N. E. Greville, Generalized Inverses: Theory and Applications (Wiley-Interscience, New York, 1974).

2. R. H. Bouldin, Generalized inverses and factorizations, in Recent Applications of Generalized Inverses, S. L. Campbell, ed., Research Notes in Mathematics 66 (Pitman, London, 1982), 233-249.

3. S. L. Campbell, The Drazin inverse of an operator, in Recent Applications of Generalized Inverses. S. L. Campbell, ed., Research Notes in Mathematics 66 (Pitman, London, 1982) 250-259.

4. S. L. Campbell and C. D. Meyer, Generalized Inverses of Linear Transformations (Pitman, London, 1979).

5. S. L. Campbell, C. D. Meyer and N. J. Rose, Applications of the Drazin inverse to linear systems of differential equations with singular constant coefficients, SIAM J. Appl. Math. 31 (1976), $411-425$.

6. S. R. Caradus, Generalized inverses and operator theory, Queen's Papers in Pure and Appl. Math. 50 (Queen's University, Kingston, Ontario, 1978).

7. M. P. Drazin, Pseudo-inverse in associative rings and semigroups, Amer. Math. Monthly 65 (1958), 506-514. $185-196$.

8. M. P. Drazin, Extremal definitions of generalized inverses, Lin. Alg. Appl. 165 (1992),

9. R. E. Harte, Spectral projections, Irish Math. Soc. Newsletter 11 (1984), 10-15.

10. R. E. Harte, Invertibility and Singularity for Bounded Linear Operators (Marcel Dekker, New York, 1988).

11. R. E. Harte, On quasinilpotents in rings, PanAm. Math. J. 1 (1991), 10-16. 
12. R. E. Hartwig, Schur's theorem and the Drazin inverse, Pacific J. Math. 78 (1978), $133-138$.

13. H. G. Heuser, Functional Analysis (Wiley, New York, 1982).

14. C. F. King, A note on Drazin inverses, Pacific J. Math. 70 (1977), 383-390.

15. J. J. Koliha, Isolated spectral points, Proc. Amer. Math. Soc., to appear.

16. D. C. Lay, Spectral properties of generalized inverses of linear operators, SIAM J. Appl. Math. 29 (1975), 103-109.

17. I. Marek and K. Žitný, Matrix Analysis for Applied Sciences, Vol. 2, Teubner-Texte zur Mathematik Band 84 (Teubner, Leipzig, 1986). 1976).

18. M. Z. Nashed, ed., Generalized Inverses and Applications (Academic Press, New York,

19. M. Z. Nashed and Y. Zhao, The Drazin inverse for singular evolution equations and partial differential equations, World Sci. Ser. Appl. Anal. 1 (1992), 441-456.

20. U. G. Rothblum, A representation of the Drazin inverse and characterizations of the index, SIAM J. Appl. Math. 31 (1976), 646-648.

21. B. Simeon, C. Führer and P. Rentrop, The Drazin inverse in multibody system dynamics, Numer. Math. 64 (1993), 521-539.

22. A. E. Taylor and D. C. Lay, Introduction to functional analysis, 2nd edition (Wiley, New York, 1980).

Department of Mathematics

UNIVERSITY of MELBOURNE

Parkville

VICTORIA 3052

Australia 